\title{
Passive Parametric Macromodeling by Using Sylvester State-Space Realizations
}

\author{
Elizabeth Rita Samuel, Luc Knockaert, and Tom Dhaene \\ Ghent University - IMinds, Gaston Crommenlaan 8 Bus 201, B-9050 Gent, Belgium, \\ elizabeth.ritasamuel, luc.knockaert, tom.dhaene@ugent.be, \\ WWW home page: http://www.sumo.intec.ugent.be/
}

\begin{abstract}
A judicious choice of the state-space realization is required in order to account for the assumed smoothness of the state-space matrices with respect to the design parameters. The direct parameterization of poles and residues may be not appropriate, due to their possible nonsmooth behavior with respect to design parameters. This is avoided in the proposed technique, by converting the a pole-residue description to a Sylvester description which is computed for each root macromodel.This technique is used in combination with suitable parameterizing schemes for interpolating a set of state-space matrices, and hence the poles and residues indirectly, in order to build accurate parametric macromodels. The key features of the present approach are first the choice of a proper pivot matrix and second, finding a well-conditioned solution of a Sylvester equation. Stability and passivity are guaranteed by construction over the design space of interest. Pertinent numerical examples validate the proposed Sylvester technique for parametric macromodeling.
\end{abstract}

Keywords: Sylvester equation, parametric macromodel, state-space matrices, interpolation.

\section{Introduction}

Multiple simulations are often required during a typical design process of electromagnetic (EM) systems, design space exploration, design optimization, and sensitivity analysis for different design parameter values (e.g., layout features). Parametric macromodels are valuable tools for efficiently and accurately performing these design activities, while avoiding new measurements or simulations at each new parameter configuration. Parameterized macromodels are multivariate models that describe the complex behavior of EM systems, typically characterized by frequency (or time) and several geometrical and physical design parameters, such as layout or substrate features. Recently, parametric macromodeling techniques able to guarantee overall stability and passivity have been proposed in $[1,3,2,4]$. Unfortunately, these methods are sensitive to issues related to the interpolation of state-space matrices [5], such as the smoothness of the state-space matrices as a function of the parameters.

The direct parameterization of poles and residues is in general not appropriate, due to their possible non-smooth behavior with respect to the design 
parameters. This is avoided in the proposed technique, where a suitable set of state-space matrices is parametric and, hence, the poles and residues indirectly. A conversion from the pole-residue description obtained by means of vector fitting (VF) to a Sylvester description is computed for each root macromodel. This avoids the direct parameterization of poles and residues, when interpolating a set of state-space matrices in order to build a parametric macromodel. The present technique is able to deal accurately with bifurcation effects of poles and residues [6].

The key features of the Sylvester realization technique are first the choice of a pivot or reference matrix and second, the obtention of a well-conditioned solution to the Sylvester equation. Since the same pivot matrix is used for all state-space realizations of the root macromodels, smooth variations of the state-space matrices with respect to the design parameters can be expected. The state-space matrices obtained from the Sylvester realization are used to obtain matrix solutions of the linear matrix inequalities (LMIs) pertaining to the positive-real or bounded-real lemma, and this information is then used to perform a passivity preserving interpolation of the state-space matrices. The computations can be carried out using the solution of LMIs or algebraic Riccati equations (AREs) to generate a descriptor state-space format that preserves positive-realness or bounded-realness. Finally, suitable interpolation schemes are used to build accurate parametric macromodels which preserve stability and passivity.

Pertinent numerical examples validate the proposed Sylvester realization technique for macromodeling based on interpolation of state-space matrices.

\section{Parametric Macromodeling}

We start with a set of passive models $\mathcal{S}_{k}, \quad k=1 \cdots N$ with given minimal realizations

$$
\mathcal{S}_{k} \equiv\left[\begin{array}{ll}
A_{k} & B_{k} \\
C_{k} & D_{k}
\end{array}\right]
$$

state space equations

$$
\begin{aligned}
& \dot{x}=A_{k} x+B_{k} u \\
& y=C_{k} x+D_{k} u
\end{aligned}
$$

and transfer functions

$$
H_{k}(s)=C_{k}\left(s I-A_{k}\right)^{-1} B_{k}+D_{k}
$$

In this paper we suppose that all realizations $\mathcal{S}_{k}$ have the same McMillan degree $n$ and number of ports $m \leq n$. This means that all $A_{k}, B_{k}, C_{k}, D_{k}$ matrices have respective sizes $n \times n, n \times m, m \times n, m \times m$. We further suppose that all matrices $A_{k}$ are Hurwitz stable i.e., all their poles are in the open left halfplane.

We aim at obtaining a generic parametric realization of the form

$$
\mathcal{S}(\mathbf{g}) \equiv\left[\begin{array}{ll}
A(\mathbf{g}) & B(\mathbf{g}) \\
C(\mathbf{g}) & D(\mathbf{g})
\end{array}\right]
$$


with vectorial parameter $\mathbf{g}$ such that the models $\mathcal{S}_{k}$ can be considered as snapshots of $\mathcal{S}(\mathbf{g})$ generated by freezing the parameter $\mathbf{g}$ at the fixed values $\mathbf{g}_{k}$. The objective is to construct a guaranteed passive macromodel $\tilde{\mathcal{S}}(\mathrm{g})$ by means of the discrete models $\mathcal{S}_{k}$, such that $\tilde{\mathcal{S}}(\mathrm{g})$ is passive, smooth and close to $\mathcal{S}(\mathrm{g})^{1}$ in some sense.

\section{State-Space Realizations For Parametric Macromodeling}

To obtain accurate parametric macromodels by interpolation of the state-space matrices, the choice of the state-space realization is fundamental.

In this section, we will discuss the well-known Gilbert realization, the balanced realization and then the proposed novel Sylvester realization, preceded by a subsection on passive parametric interpolation.

\subsection{Gilbert Realization}

The minimal state-space realization problem for linear time invariant (LTI) systems was first stated by Gilbert [7], who gave an algorithm for transforming a transfer function into a system of differential equations. The Gilbert approach is based on partial-fraction expansions.

$$
H(s)=R_{0, \boldsymbol{p}_{k}}+\sum_{n=1}^{N} \frac{R_{n, \boldsymbol{p}_{k}}}{s-z_{n, \boldsymbol{p}_{k}}}
$$

where $R_{n, \boldsymbol{p}_{k}}$ and $s-z_{n, \boldsymbol{p}_{k}}$ are respectively the model residues and poles, with $R_{0, \boldsymbol{p}_{k}}$ being the direct coupling constant. The poles and the residues are stamped directly in the $A(\boldsymbol{p})$ and $C(\boldsymbol{p})$ matrices using the Gilbert realization [7]. It is wellknown that model poles and residues are very sensitive to even small variations of the design parameters, resulting in quite irregular variations of each pole in the design space. Since poles and residues may present a highly non-smooth behavior with respect to the design parameters, achieving a reasonable accuracy in parametric macromodels built by interpolation of state-space matrices becomes difficult, due to the fact that pole and residue trajectories as a function of $\boldsymbol{p}$ are not well defined.

\subsection{Balanced Realization}

A minimal and stable realization is called balanced, if the controllability and observability Gramians are equal and diagonal [8]. Every minimal system can be brought into balanced form. The balanced realization can be implemented using the Matlab function balreal. This routine uses the eigendecomposition

${ }^{1}$ The exact generic realization $\mathcal{S}(\mathbf{g})$ is analytically unknown in the sense that for each new value of $\mathbf{g}$ an oracle (or black-box function) has to be consulted. 
of the product of the observability and controllability Gramians to construct the balancing transformation matrix.

The most interesting properties of balanced realizations is associated with the uniqueness property of the balancing transformation [9]. When the eigenvalues (real and nonnegative) of the product of the controllability and observability Gramians, are distinct, then the balancing transformation matrix is unique. If, on the other hand, two or more eigenvalues are repeated, then their corresponding eigenvectors can be rotated arbitrarily in the corresponding eigenspace. Thus as stated in $[5,9]$, uniqueness is guaranteed up to a sign and it may affect the smoothness of the state-space matrices as functions of the design parameters.

\subsection{Passive Interpolation of LTI Systems}

Passivity is an important property to satisfy because stable, but not passive macromodels can produce unstable systems when connected to other stable, even passive, loads.

As a first approach we opt for straightforward passive interpolation. Since each macromodel $\mathcal{S}_{k}$ is passive, by the positive real lemma [10] we know that this is the case if there exists a positive definite symmetric matrix $P_{k}$ such that the Linear Matrix Inequality (LMI) [11]

$$
\mathcal{L}_{k}=\left[\begin{array}{cc}
A_{k}^{T} P_{k}+P_{k} A_{k} & P_{k} B_{k}-C_{k}^{T} \\
B_{k}^{T} P_{k}-C_{k} & -D_{k}-D_{k}^{T}
\end{array}\right] \leq 0
$$

is satisfied. Now consider a positive interpolation kernel [12] $\mathcal{K}\left(\mathbf{g}_{k}, \mathbf{g}\right)=\mu_{k}(\mathbf{g})$ satisfying

$$
\mu_{k}(\mathbf{g}) \geq 0, \quad \mu_{k}\left(\mathbf{g}_{l}\right)=\delta_{k, l}
$$

It is clear that the interpolatory parametric LMI

$$
\mathcal{L}(\mathbf{g})=\sum_{k=1}^{N} \mu_{k}(\mathbf{g}) \mathcal{L}_{k}
$$

is semi-negative definite, and hence if we parameterize all entries of the $P_{k} A_{k}$, $P_{k} B_{k}, C_{k}, D_{k}, P_{k}$ matrices as

$$
\begin{aligned}
P(\mathbf{g}) A(\mathbf{g}) & =\sum_{k=1}^{N} \mu_{k}(\mathbf{g}) P_{k} A_{k} \\
P(\mathbf{g}) B(\mathbf{g}) & =\sum_{k=1}^{N} \mu_{k}(\mathbf{g}) P_{k} B_{k} \\
C(\mathbf{g}) & =\sum_{k=1}^{N} \mu_{k}(\mathbf{g}) C_{k} \\
D(\mathbf{g}) & =\sum_{k=1}^{N} \mu_{k}(\mathbf{g}) D_{k}
\end{aligned}
$$




$$
P(\mathbf{g})=\sum_{k=1}^{N} \mu_{k}(\mathbf{g}) P_{k}
$$

it is seen by inspection that the parametric realization

$$
\tilde{\mathcal{S}}(\mathbf{g}) \equiv\left[\begin{array}{ll}
A(\mathbf{g}) & B(\mathbf{g}) \\
C(\mathbf{g}) & D(\mathbf{g})
\end{array}\right]
$$

thus obtained is passive with LMI solution matrix $P(\mathbf{g})$.

\subsection{Uniform Approach by Sylvester Equations}

The issues with the passive parametric interpolation procedure when using the Gilbert and balanced realizations are twofold. First, there are 5 interpolation equations (10) to be satisfied. Second, and most important, although the interpolation technique yields (by construction) the discrete macro-models $\mathcal{S}_{k}$ for $\mathbf{g}=$ $\mathbf{g}_{k}$, it is not at all sure that the interpolated matrices $A(\mathbf{g}), B(\mathbf{g}), C(\mathbf{g}), D(\mathbf{g}), P(\mathbf{g})$ will behave smoothly between the nodes $\mathbf{g}_{k}$. The reason for this is that minimal realizations are all equivalent modulo a similarity transformation, i.e., two realizations related by

$$
\left[\begin{array}{ll}
\tilde{A} & \tilde{B} \\
\tilde{C} & \tilde{D}
\end{array}\right]=\left[\begin{array}{cc}
X^{-1} A X & X^{-1} B \\
C X & D
\end{array}\right]
$$

where $X$ is any nonsingular matrix, yield the same transfer function

$$
H(s)=C(s I-A)^{-1} B+D=\tilde{C}(s I-\tilde{A})^{-1} \tilde{B}+\tilde{D}
$$

In order to install uniformity we propose the state-space feedback realization

$$
\begin{aligned}
\dot{x} & =\mathbf{A} x+\mathbf{B}_{k} v \\
y & =\hat{\mathbf{C}}_{k} x+D_{k} v \\
v & =-\mathbf{F} x+u
\end{aligned}
$$

where $\mathbf{A}$ is a fixed $n \times n$ pivot matrix and $\mathbf{F}$ is a fixed $m \times n$ state-space feedback matrix. This realization can be written as

$$
\mathcal{R}_{k} \equiv\left[\begin{array}{cc}
\mathbf{A}-\mathbf{B}_{k} \mathbf{F} & \mathbf{B}_{k} \\
\hat{\mathbf{C}}_{k}-D_{k} \mathbf{F} & D_{k}
\end{array}\right]=\left[\begin{array}{cc}
\mathbf{A}-\mathbf{B}_{k} \mathbf{F} \mathbf{B}_{k} \\
\mathbf{C}_{k} & D_{k}
\end{array}\right]
$$

For $\mathcal{R}_{k}$ and $\mathcal{S}_{k}$ to be equivalent, we need the existence of nonsingular matrices $X_{k}$ such that

$$
\begin{aligned}
\mathbf{A}-\mathbf{B}_{k} \mathbf{F} & =X_{k}^{-1} A_{k} X_{k} \\
\mathbf{B}_{k} & =X_{k}^{-1} B_{k} \\
\mathbf{C}_{k} & =C_{k} X_{k}
\end{aligned}
$$


Eliminating (19) from (18) we obtain the Sylvester equation

$$
A_{k} X_{k}-X_{k} \mathbf{A}+B_{k} \mathbf{F}=0
$$

for the unknown matrix $X_{k}$. We need the following

Theorem 1. The Sylvester equation (21) has a unique nonsingular solution $X_{k}$ provided the pair $\left(A_{k}, B_{k}\right)$ is controllable, the pair $(\mathbf{A}, \mathbf{F})$ is observable, and the intersection of the eigenspectra of $A_{k}$ and $\mathbf{A}$ is empty.

Proof. See $[13,14]$.

The Sylvester equations are routinely solved by the Matlab function lyap.

Remark 1. The Sylvester realizations $\mathcal{R}_{k}$, given the pivot matrix $\mathbf{A}$ and feedback matrix $\mathbf{F}$, are all unique by construction. For the choice of $\mathbf{A}$ we can take a block-diagonal or block-Jordan matrix[14] which never shares eigenvalues with any of the $A_{k}$ matrices. This can be accomplished by choosing the eigenvalues of $\mathbf{A}$ close to the imaginary axis (see also the numerical simulations). The choice of $\mathbf{F}$ is subject to the requirement that the pair $(\mathbf{A}, \mathbf{F})$ has to be observable. In some cases such as the Gilbert [7] or Vector Fitting [15] realization, all matrices $B_{k}$ are equal, and then a judicious choice for $\mathbf{F}$ is $\mathbf{F}=B_{k}^{T}$. More generally speaking, $\mathbf{F}$ can be chosen quite freely, or its choice can be imbedded in the overall Sylvester algorithm [16]. Next, we parameterize the new LMI's

$$
\left[\begin{array}{cc}
\left(\mathbf{A}-\mathbf{B}_{k} \mathbf{F}\right)^{T} \tilde{P}_{k}+\tilde{P}_{k}\left(\mathbf{A}-\mathbf{B}_{k} \mathbf{F}\right) & \tilde{P}_{k} \mathbf{B}_{k}-\mathbf{C}_{k}^{T} \\
\mathbf{B}_{k}^{T} \tilde{P}_{k}-\mathbf{C}_{k} & -D_{k}-D_{k}^{T}
\end{array}\right] \leq 0
$$

as in Section 3.3, four last equations of (10), i.e.,

$$
\begin{aligned}
\tilde{P}(\mathbf{g}) \mathbf{B}(\mathbf{g}) & =\sum_{k=1}^{N} \mu_{k}(\mathbf{g}) \tilde{P}_{k} \mathbf{B}_{k} \\
\mathbf{C}(\mathbf{g}) & =\sum_{k=1}^{N} \mu_{k}(\mathbf{g}) \mathbf{C}_{k} \\
D(\mathbf{g}) & =\sum_{k=1}^{N} \mu_{k}(\mathbf{g}) D_{k} \\
\tilde{P}(\mathbf{g}) & =\sum_{k=1}^{N} \mu_{k}(\mathbf{g}) \tilde{P}_{k}
\end{aligned}
$$

The first equation of (10) has no counterpart in equations (23), since it is easy to show that

$$
\tilde{P}(\mathbf{g})[\mathbf{A}-\mathbf{B}(\mathbf{g}) \mathbf{F}]=\sum_{k=1}^{N} \mu_{k}(\mathbf{g}) \tilde{P}_{k}\left[\mathbf{A}-\mathbf{B}_{k} \mathbf{F}\right]
$$


Finally, the parametric Sylvester realization is then simply

$$
\mathcal{R}(\mathbf{g}) \equiv\left[\begin{array}{cc}
\mathbf{A}-\mathbf{B}(\mathbf{g}) \mathbf{F} & \mathbf{B}(\mathbf{g}) \\
\mathbf{C}(\mathbf{g}) & D(\mathbf{g})
\end{array}\right]
$$

This also implies that we can track the pole trajectories of the parametric system easily as the eigenvalues of the matrix $\mathbf{A}-\mathbf{B}(\mathbf{g}) \mathbf{F}$, which depends only on the parameterization of $\mathbf{B}(\mathbf{g})$, i.e.

$$
\mathbf{B}(\mathbf{g})=\left(\sum_{k=1}^{N} \mu_{k}(\mathbf{g}) \tilde{P}_{k}\right)^{-1} \sum_{k=1}^{N} \mu_{k}(\mathbf{g}) \tilde{P}_{k} \mathbf{B}_{k}
$$

Remark 2. Note that, even if passivity is not required, the Sylvester realizations $\mathcal{R}_{k}$ can be very useful for parameterization. Suppose the interpolation kernel $\mathcal{K}\left(\mathbf{g}_{k}, \mathbf{g}\right)=\mu_{k}(\mathbf{g})$ is not necessarily positive, but satisfies partition of $u_{n i t y}^{2}$, i.e.,

$$
\sum_{k} \mu_{k}(\mathbf{g})=1, \quad \mu_{k}\left(\mathbf{g}_{l}\right)=\delta_{k, l}
$$

Then it is clear that the interpolation procedure

$$
\begin{aligned}
\mathbf{B}(\mathbf{g}) & =\sum_{k=1}^{N} \mu_{k}(\mathbf{g}) \mathbf{B}_{k} \\
\mathbf{C}(\mathbf{g}) & =\sum_{k=1}^{N} \mu_{k}(\mathbf{g}) \mathbf{C}_{k} \\
D(\mathbf{g}) & =\sum_{k=1}^{N} \mu_{k}(\mathbf{g}) D_{k}
\end{aligned}
$$

is a very simple way to generate a parametric macromodel.

\section{Solving LMI's}

\subsection{Convex programming}

LMI's such as (7) are convex formulations and can always be solved by convex optimization [17], without needing ARE solvers and/or Hamiltonian matrices. A standard trick in convex optimization is to transform the problem that must be solved into an equivalent problem, which is in a standard form that can be solved by a generic solver. Recently developed parser-solvers, such as YALMIP [18], CVX [19], CVXMOD [20], and Pyomo [21] automate this reduction process. A

\footnotetext{
${ }^{2}$ Note that multilinear interpolation satisfies both positivity and partition of unity.
} 
general approach called disciplined convex programming $[22,23]$ has emerged as an effective methodology for organizing and implementing parser-solvers for convex optimization. In disciplined convex programming, the user combines built-in functions in specific, convexity-preserving ways. The constraints and objective must also follow certain rules. As long as the user conforms to these requirements, the parser can easily verify the convexity of the problem and automatically transform it to a standard form for transfer to the solver. Note that the parser-solvers CVX (which runs in Matlab ) and CVXMOD (Python) use the disciplined convex programming approach. For example, in our case we solve LMI (7) by means of the CVX code

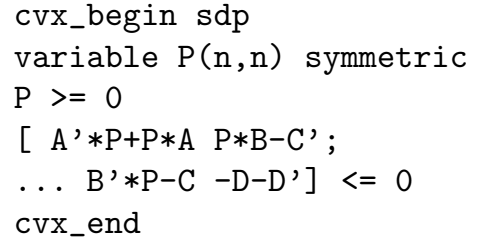

\subsection{Riccati equations}

An LMI of the form (7), i.e.,

$$
\left[\begin{array}{c}
A^{T} P+P A P B-C^{T} \\
B^{T} P-C-D-D^{T}
\end{array}\right] \leq 0
$$

can be solved by converting to the Lur'e equations [24]

$$
\begin{aligned}
A^{T} P+P A & =-Q^{T} Q \\
P B-C^{T} & =-Q^{T} W \\
W^{T} W & =D+D^{T}
\end{aligned}
$$

In the case $D+D^{T}>0$, the matrices $W$ and $Q$ can be eliminated, yielding the algebraic Riccati equation

$$
A^{T} P+P A+\left(P B-C^{T}\right)\left(D+D^{T}\right)^{-1}\left(B^{T} P-C\right)=0
$$

Hence, if $D+D^{T}>0$, the system is positive real if the algebraic Riccati equation (31) has a stabilizing solution $P[25]$.

If $D+D^{T}$ is only semi-positive definite, i.e., $\operatorname{det}\left(D+D^{T}\right)=0$, the situation is much more complicated and the approaches in $[26,27]$ may provide solutions. However, the Riccati approach may also be rescued by means of the following theorem:

Theorem 2. Frequency inversion theorem : Let $H(s)=C\left(s I_{n}-A\right)^{-1} B+D$ be minimal and positive-real with A Hurwitz. Then $G(s)=\tilde{C}\left(s I_{n}-\tilde{A}\right)^{-1} \tilde{B}+\tilde{D}$ with

$$
\begin{aligned}
& \tilde{A}=A^{-1} \quad \tilde{B}=A^{-1} B \\
& \tilde{C}=-C A^{-1} \quad \tilde{D}=D-C A^{-1} B
\end{aligned}
$$

is also positive real and admits the same $P$ matrix as $H(s)$. 
Proof. It is straightforward to see that when $A$ is Hurwitz, then $A^{-1}$ is also Hurwitz and vice versa. Also, it is simple to see by substitution that $G(s)=$ $H(1 / s)$. By positive-realness, $H(s)$ admits a factorization [24] :

$$
H(s)+H(-s)^{T}=M(-s)^{T} M(s) \quad \forall s \in \mathcal{C}_{+}
$$

Since the mapping $s \mapsto 1 / s$ is one-to-one in (extended) $\mathcal{C}_{+}$, it follows that

$$
\begin{aligned}
G(s)+G(-s)^{T} & =H(1 / s)+H(-1 / s)^{T} \\
& =M(-1 / s)^{T} M(1 / s) \quad \forall s \in \mathcal{C}_{+}
\end{aligned}
$$

In other words $G(s)$ is positive-real. To prove it admits the same $P$ as $H(s)$ we write the Lur'e equations

$$
\begin{aligned}
A^{T} P+P A & =-Q^{T} Q \\
P B-C^{T} & =-Q^{T} W \\
D+D^{T} & =W^{T} W
\end{aligned}
$$

Define $\mathcal{Q}=-Q A^{-1}$ and $\mathcal{W}=W-Q A^{-1} B$. It is easy to see that

$$
\tilde{A}^{T} P+P \tilde{A}=-\mathcal{Q}^{T} \mathcal{Q}
$$

Also

$$
-\mathcal{Q}^{T} \mathcal{W}=A^{-T}\left[Q^{T} W-Q^{T} Q A^{-1} B\right]=P \tilde{B}-\tilde{C}^{T}
$$

and finally

$$
\tilde{D}+\tilde{D}^{T}=\mathcal{W}^{T} \mathcal{W}
$$

Note that $\tilde{D}=H(0)$ and hence Theorem 2 maps the positive-realness problem from $s=\infty$ to $s=0$. Of course it could be that both $H(\infty)+H(\infty)^{T}$ and $H(0)+H(0)^{T}$ are singular, in which case the approach in [26] may provide solution.

\section{$5 \quad$ Numerical Simulations}

In the following examples, we show the importance of the realization issue, and validate the proposed Sylvester approach, by comparing them with the standard Gilbert and balanced realizations.

\subsection{Two coupled microstrip with Variable Length}

Two coupled microstrips can be modeled starting from per-unit-length parameters. The cross section is shown in Fig.1.

Figure. 1 shows its cross section. The length $L$ are considered as variable parameters in addition to frequency. Their corresponding ranges are shown in Table 1. 


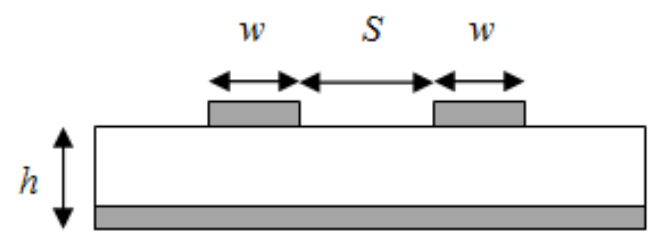

Fig. 1. Two coupled microstrip line.

Table 1. PARAMETERS OF THE COUPLED MICROSTRIPS

\begin{tabular}{lll}
\hline Parameter & Min & Max \\
\hline Frequency (freq) & $20 \mathrm{MHz}$ & $8 \mathrm{GHz}$ \\
Length $(L)$ & $2.5 \mathrm{~cm}$ & $3 \mathrm{~cm}$ \\
\hline
\end{tabular}

The scattering parameters were obtained over a validation grid of $200 \times 11$ samples, for frequency and length respectively. We have built root macromodels for 6 values of the spacing by means of VF, each with an order 11 .

As described in Section 3.4, a pivot matrix and a feedback matrix is chosen such that a well-conditioned solution is obtained for the Sylvester equation (21).

Also, since the eigenvalues of the pivot matrix and those of the root macromodels obtained from Gilbert realization must not be the same, we choose the poles very close to the imaginary axis as shown in Fig.2. The feedback matrix is chosen as column vectors of 1's, 2's and 0's similar to VF technique. A similarity transformation is then performed using the Sylvester solution to obtain the state-space matrices of the Sylvester realization.

Next the realizations are converted to a passive descriptor state-space form using LMI (7) as described in Section 4 with the help of CVX. Finally, a bivariate macromodel is obtained by linear interpolation of the corresponding state-space matrices using the Sylvester realization as shown in Fig.3.

The maximum absolute error over the validation grid for the parametric macromodel of the scattering matrix is bounded by $-56 d B$. Note that a very good agreement is obtained between the original data and the proposed parametric macromodeling technique. The parametric macromodel captures the behavior of the system very accurately over the entire range of the length.

Figure.4 shows that direct parameterization of the poles should be avoided due to potentially non-smooth behavior with respect to the design parameters with Gilbert realization.

In Figure. 5 it is shown that the maximum absolute error is very small for the Sylvester but it is not satisfactory for the Gilbert and balanced real realization. 


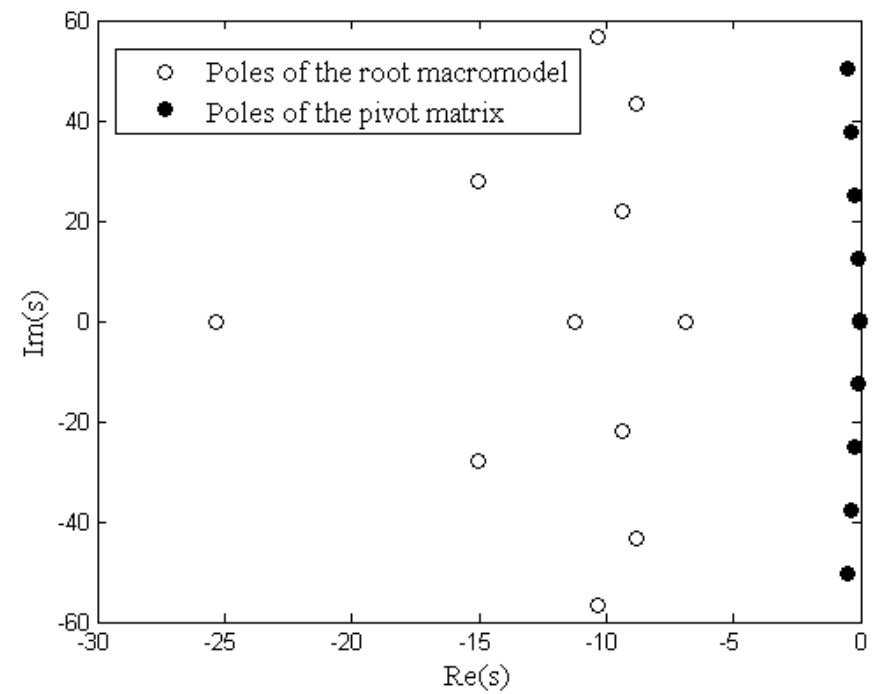

Fig. 2. Eigenvalues of the pivot matrix and the root macromodels obtained from Gilbert realization.

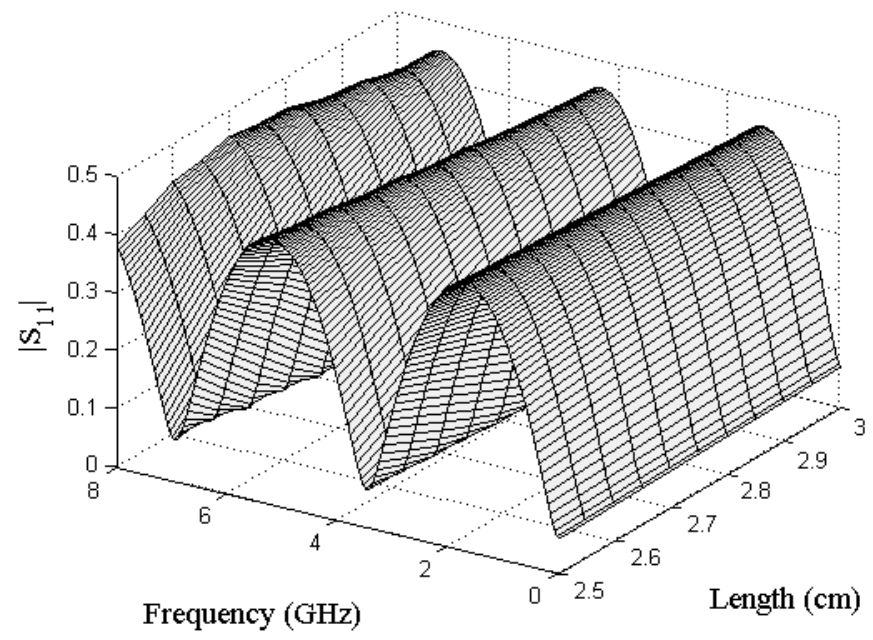

Fig. 3. Magnitude of the bivariate macromodel $S_{11}(s, L)$ (Sylvester realization for each root macromodel). 


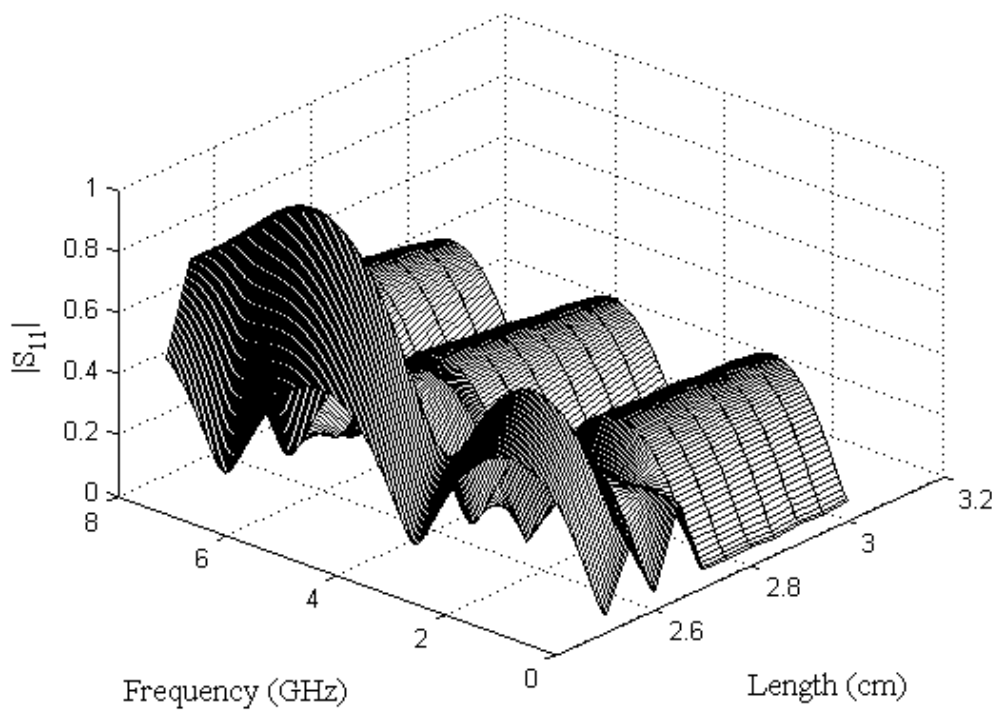

Fig. 4. Magnitude of the bivariate macromodel $S_{11}(s, L)$ (Gilbert realization form for each root macromodel).

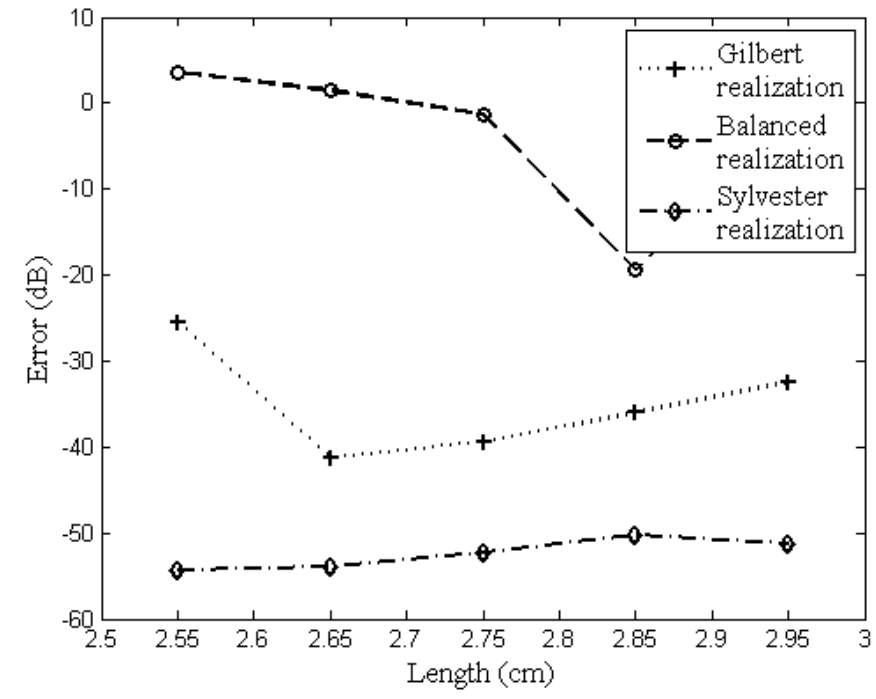

Fig. 5. CM: Absolute error comparison for the different realizations. 


\subsection{Hairpin bandpass microwave filter}

In this example, a hairpin bandpass filter with the layout shown in Figure.6 is modeled. The relative permittivity of the substrate is 9.9 , and its thickness is equal to $0.635 \mathrm{~mm}$.

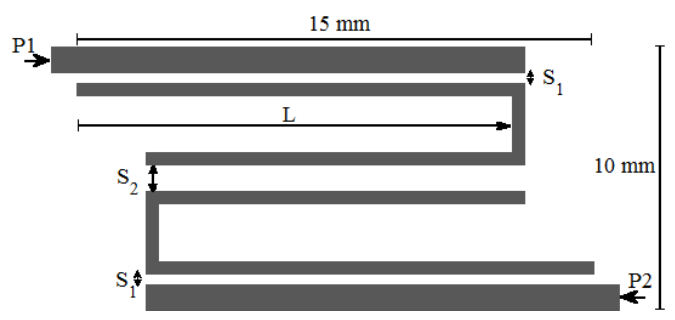

Fig. 6. Layout of the folded stub notch filter.

The spacing $S_{1}$ and the length $L$ of the stub are chosen as design variables in addition to frequency. Their corresponding ranges are shown in Table 2.

Table 2. PARAMETERS OF THE HAIRPIN BANDPASS MICROWAVE FILTER

\begin{tabular}{lll}
\hline Parameter & Min & Max \\
\hline Frequency (freq) & $1.5 \mathrm{GHz}$ & $3.5 \mathrm{GHz}$ \\
Length $(L)$ & $12 \mathrm{~mm}$ & $12.5 \mathrm{~mm}$ \\
Spacing $\left(S_{1}\right)$ & $0.27 \mathrm{~mm}$ & $0.32 \mathrm{~mm}$ \\
\hline
\end{tabular}

The scattering parameters have been computed by means of the advanced design system $(\mathrm{ADS})$ over a grid of $11 \times 7$ samples, for length and spacing respectively. We have built root macromodels for $6 \times 4$ values of the length and spacing respectively by means of VF, each with an order 13 . Next the realization approaches as described in Section 3.3 is used to obtain Sylvester realized statespace form for each root macromodel. Then the realizations are converted to a passive descriptor state-space form using LMI (7) as described in Section 4 with the help of CVX.

Finally, a trivariate macromodel is obtained by multilinear interpolation of the corresponding state-space matrices as shown in Figure.7.

The maximum absolute error over the validation grid for the parametric macromodel of the scattering matrix is bounded by $-58 \mathrm{~dB}$. It can be noted that a very good agreement is obtained between the original data and the proposed parametric macromodeling technique. The parametric macromodel captures the behavior of the system very accurately over the entire design space.

Figure. 8 shows the parametric macromodel using balanced real realization. It is seen by comparing with Figure. 7 that the behavior is very erratic. 


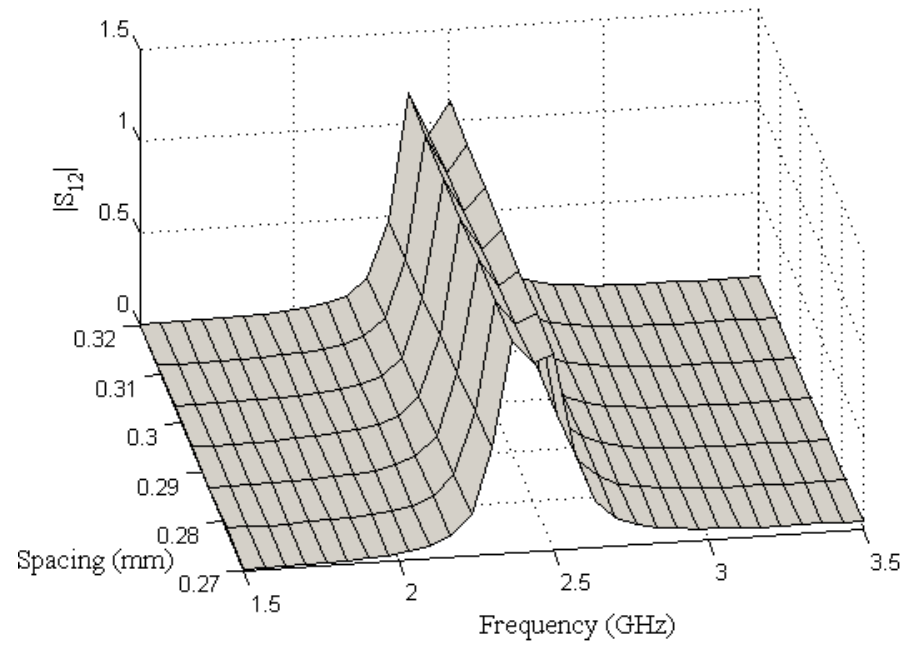

Fig. 7. Magnitude of the trivariate macromodel $S_{12}(s, L, S)$ for $L=12.05 \mathrm{~mm}$ (Sylvester realization for each root macromodel).

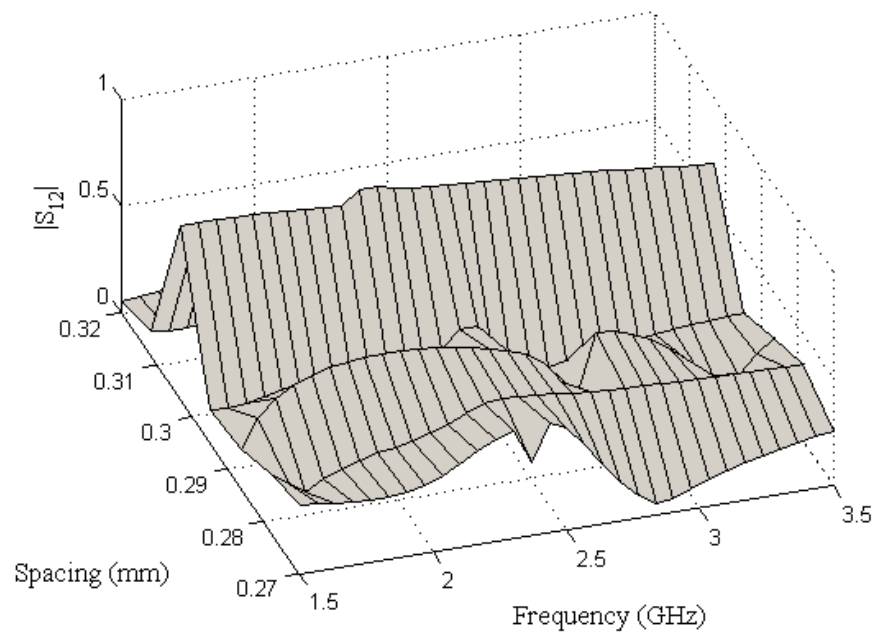

Fig. 8. Magnitude of the trivariate macromodel $S_{12}(s, L, S)$ for $L=12.05 \mathrm{~mm}$ (Balanced realization for each root macromodel). 
For the hairpin filter it can be also noted from the Figure.9 that the maximum absolute error is very small for the Sylvester realization but it is not satisfactory for Gilbert realization and balanced real realization.

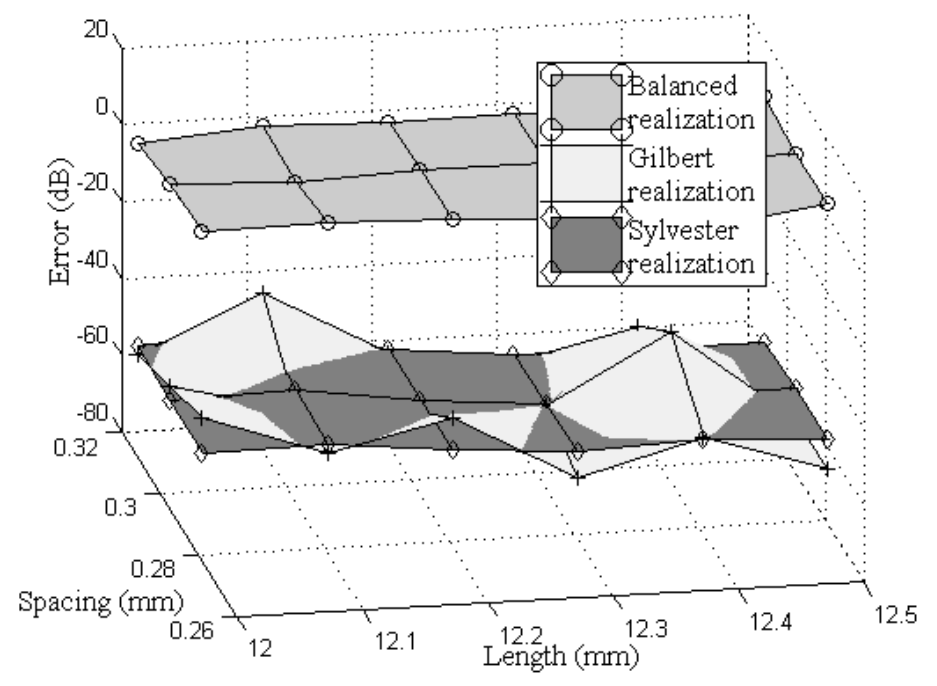

Fig. 9. Absolute error comparison for the different realizations.

\section{Conclusion}

This paper proposes a novel state-space realization for parametric macromodeling based on interpolation of state-space matrices. A good choice of the statespace realization is required to account for the generally assumed smoothness of the state-space matrices with respect to the parameters. Suitable interpolation schemes along with Sylvester realization are used to interpolate a set of root state-space matrices in order to build accurate parametric macromodels. There are two essential aspects for this novel realization: 1) to find a proper pivot matrix and 2) to obtain a well-conditioned solution for a Sylvester equation. The numerical examples and related comparison results show that the proposed Sylvester realization provides very accurate parametric macromodel with a low computational cost. The properties of the system like stability and passivity can be preserved with the help of LMIs and by the use of proper interpolation schemes. 


\section{Acknowledgment}

This research has been funded by the Research Foundation Flanders (FWO) and the Interuniversity Attraction Poles Programme BESTCOM initiated by the Belgian Science Policy Office.

\section{References}

1. F. Ferranti, L. Knockaert, and T. Dhaene, "Guaranteed passive parameterized admittance-based macromodeling," IEEE Transactions on Advanced Packaging, vol. 33, no. 3, pp. 623 629, Aug. 2010.

2. F. Ferranti, L. Knockaert, and T. Dhaene,"Parameterized S-parameter based macromodeling with guaranteed passivity," IEEE Microwave and Wireless Component Letters, vol. 19, no. 10, pp. 608610, Oct. 2009.

3. F. Ferranti, L. Knockaert, T. Dhaene, and G. Antonini, Passivity preserving parametric macromodeling for highly dynamic tabulated data based on Lure equations, IEEE Transactions on Microwave Theory and Techniques, vol. 58, no. 12, pp. 3688 3696, Dec. 2010.

4. P. Triverio, M. Nakhla, and S. Grivet-Talocia, "Passive parametric modeling of interconnects and packaging components from sampled impedance, admittance or scattering data," Electronic System-Integration Technology Conference, pp. 16, Sept. 2010.

5. J. De Caigny, J. F. Camino, and J. Swevers, "Interpolating model identication for siso linear parameter-varying systems," Mechanical Systems and Signal Processing, vol. 23, no. 8, pp. 23952417, 2009.

6. E. R. Samuel, L. Knockaert, F. Ferranti, T. Dhaene, "Guaranteed Passive Parameterized Macromodeling by Using Sylvester State-Space Realizations," IEEE Transactions on Microwave Theory and Techniques, vol.61, no.4, pp.1444-1454, April 2013.

7. E. G. Gilbert, "Controllability and observability in multi-variable control systems," SIAM Journal on Control, vol. 1, no. 2, pp. 128151, 1963.

8. B. Moore, "Principal component analysis in linear systems: Controllability, observability, and model reduction," IEEE Transactions Automatic Control, vol. 26, no. 1, pp. 1731, Feb. 1981.

9. M. Lovera and G. Mercere, "Identification for gain-scheduling: a balanced subspace approach," American Control Conference, 2007, pp. 858 863, July 2007.

10. B.D.O. Anderson and S. Vongpanitlerd, Network Analysis and Synthesis. PrenticeHall, Englewood Cliffs, NJ., 1973.

11. F. Ferranti, L. Knockaert, T. Dhaene, and G. Antonini, Parametric macromodeling for S-parameter data based on internal nonexpansivity, International Journal of Numerical Modelling: Electronic Networks, Devices and Fields, vol. 26, no. 1, pp. 1527, 2013.

12. F. Ferranti, L. Knockaert, and T. Dhaene, Passivity-preserving parametric macromodeling by means of scaled and shifted state-space systems, IEEE Transactions on Microwave Theory and Techniques, vol. 59, no. 10, pp. 2394 2403, Oct. 2011.

13. E. De Souza and S. P. Bhattacharyya, "Controllability, observability and the solution of AX-XB = C," Lin.Alg. \& Appl., no. 39, pp. 167-188, 1981.

14. A. Varga, "Robust pole assignment via Sylvester equation based state feedback parametrization," Proc. IEEE International Symposium on Computer-Aided Control System Design, pp. 13-18, 2000. 
15. B. Gustavsen and A. Semlyen, "Rational approximation of frequency domain responses by vector fitting", IEEE Trans. Power Delivery, vol. 14, no. 3, pp. 1052 1061, July 1999.

16. J. Carvalho, K. Datta, and Y. Hong, "A new block algorithm for full-rank solution of the Sylvester-observer equation," IEEE Trans. Automatic Control, vol. 48, no. 12, pp. 2223-2228, Dec. 2003.

17. S. Boyd and L. Vandenberghe, Convex Optimization, Cambridge Univ. Press, Cambridge, U.K., 2004. Available at http://www.math.nus.edu.sg/ mattohkc/sdpt3.html

18. J. Löfberg, " YALMIP: A toolbox for modeling and optimization in MATLAB," Proc. CACSD Conf., Taipei, Taiwan, 2004. Available: http://control. ee.ethz.ch/ joloef/yalmip.php

19. M. Grant and S. Boyd, "CVX: Matlab software for disciplined convex programming (web page and software)," July 2008. Available: http://www. stanford.edu/ boyd/cvx/

20. J. E. Mattingley and S. Boyd, "CVXMOD: Convex optimization software in Python (web page and software)," Aug. 2008. Available: http://cvxmod.net/

21. N. L. Benavides, R. D. Carr, and W. E. Hart, "Python optimization modeling objects (Pyomo)," in Proc. INFORMS Computing Society Conf., 2009. Available: https://software.sandia.gov/trac/pyutilib/export/30/trunk/doc/ pyomo.pdf

22. M. Grant, S. Boyd, and Y. Ye, "Disciplined convex programming," in Global Optimization: From Theory to Implementation (Nonconvex Optimization and Its Applications), L. Liberti and N. Maculan, Eds. New York: Springer Science and Business Media, pp.155-210, 2006.

23. J. Mattingley and S. Boyd, "Real-time convex optimization in signal processing," IEEE Signal Processing Magazine, vol. 27 , no. 3, pp. 50-61, 2010.

24. R. F. Curtain, "Old and new perspectives on the positive-real lemma in systems and control theory," Z. Angew. Math. Mech., vol. 79, no. 9, pp.579-590, 1999.

25. S. Boyd, L. El Ghaoui, E. Feron, and V. Balakrishnan, Linear matrix inequalities in system and control theory. SIAM Studies in Applied Mathematics, 15. Society for Industrial and Applied Mathematics (SIAM), Philadelphia, PA, 1994.

26. H. Weiss, Q. Wang, and J. L. Speyer, "System characterization of positive real conditions," IEEE. Trans. Autom. Contr., vol. 39, no. 3, pp.540-544, 1994.

27. L. Knockaert, T. Dhaene, F. Ferranti, D. De Zutter, Model order reduction with preservation of passivity, non-expansivity and Markov moments, Systems $\&$ Control Letters, vol 60, no. 1, Jan. 2011, pp. 53-61. 